\title{
Sublimation versus fragmentation in matrix-assisted laser desorption
}

\author{
A. Vertes \\ Department of Chemistry, University of Antwerp (UIA), Universiteitsplein 1, 2610 Wilrijer, Belgium
}

and

R.D. Levine

The Fritz Haber Research Center for Molecular Dynamics, The Hebrew University, Jerusalem 91904, Israel

Received 10 April 1990; in final form 17 May 1990

\begin{abstract}
A kinematic model for the laser-induced introduction into the gas phase of large molecules embedded in a host matrix is discussed. The desorption of undegraded large molecules is argued to be a kinetic effect due to potentially rapid destruction of the host matrix. Two separate mechanisms are considered, sublimation and fragmentation of the matrix. Due to the choice of hosts, sublimation is more typical of experiments using UV laser pulses. In general, sublimation is found to be more efficient because it can turn itself off. The matrix destruction competes with host-guest vibrational energy transfer. The energy content of the guest can be reduced by working at low volume concentrations, special choice of host matrices and using an initially cold sample.
\end{abstract}

\section{Introduction}

Large organic and biomolecules embedded in a host matrix can be volatilized [1,2] upon rapid laser irradiation [3]. Even exceedingly high molecular weight compounds (e.g. proteins) can, under suitable condition, be introduced intact into the gas phase [4]. A (partial) destruction of the host matrix is clearly necessary for such a desorption. At somc timc during the process, the energy density in the lattice must therefore be fairly high. This Letter considers the possible mechanism whereby labile molecules emerge without thermal degradation from within such a hot lattice. The essential ingredient in our proposed scheme is that under suitable but realistic experimental conditions it is possible to achieve a volatilization of the matrix on a time short compared to that required for thermal equilibration of the guest molecules with the matrix.

Experimental descriptions of optimal conditions $[5,6]$ clearly suggest that there is more than one route to volatilization. Thus, lasers of quite different characteristics have been used (e.g. shorter pulse lasers in the UV versus longer pulse lasers in the infrared). Host matrices of quite different properties are found suitable for different laser wavelengths, etc. Indeed, we shall argue for two distinct mechanisms which we shall term "surface evaporation" and "volume evaporation", respectively. By using kinetic equations in reduced time (in terms of the laser pulse width), we shall however be able to treat both on equal footing. For typical laser and material properties we find that surface evaporation is the dominant mechanism for the high absorbing, low sublimation temperature matrices used in UV laser desorption [7]. The volume heating or "thermal stress" route is more important for typical conditions in experiments using the $\mathrm{CO}_{2}$ laser (e.g. refs. [1,5] ). We find that the latter experiments, for realistic experimental parameters often yield gas phase guest molecules of higher energy content. Such molecules would isomerize/ dissociate unless they are cooled down (e.g. by being swept in a cold supersonic beam [1]). While the $\mathrm{CO}_{2}$ laser experiments have inherently longer time scales due to their typical pulse width, it should still prove possible to desorb cold guest molecules if a host ma- 
trix of sufficiently low critical stress is used.

The thermally induced stress [8] leads to a fragmentation of the matrix. Using the maximum entropy formalism we shall argue that the fragments are predominantly small clusters of primarily undissociated host molecules. The guest molecules are then taken to be desorbed upon the attainment of critical stress. In the surface evaporation route, the guest molecules desorb when their environment sublimates.

In most respects the model is as simple as possible and many important aspects are glossed over in that they are deemed not crucial to the essence of the phenomena. One such aspect are energy gradients. We consider a model in which the energy absorbed from the laser is uniformly distributed within a "hot spot". There are many reasons why this is not the case. Obvious ones include the exponential decline of laser flux with penetration depth and heat conduction. A less obvious one is the importance of defects for volume evaporation. Inherently, due to their method of preparation, the matrices are far from perfect. In addition, defects are often intentionally introduced, e.g. by the incorporation of fine metallic particles. These have both high localized absorption in the infrared and high coefficients of thermal expansion. For these and other reasons the energy densities we compute should be regarded as representing averages over regions large in respect to host molecular dimensions. In the future it will be worthwhile to remove the assumed uniformity.

In its essence our approach is in the same vein as in an earlier simplistic description of laser-induced desorption from surfaces. There the competition was between energy transfer to a physisorbed molecule and the rupture of the physisorption bond. Here it is the rupture of the matrix that provides the time scale. In other words, the competition is between energy transfer to guest biomolecule and the mechanism of destruction of the host matrix. Because the guest molecule is embedded in the matrix, it will get warmer but, we suggest, less so than might appear at first glance. To estimate the pumping of energy into the host we use the most conservative route. Specifically, we allow hydrogen bonding between guest and host. The $\mathrm{H}$ bond being stronger than a van der Waals (physisorption) bond is of a higher frequency and hence serves less as a bottleneck. We also recognize that the biomolecule is surrounded by host mole- cules and so is pumped from all directions. Even so, we find that a competition with matrix volatization is possible. To see why, take sublimation as an example. As the lattice gets warmer, the rate of evaporation increases exponentially and for molecular crystals of low sublimation temperature, serves to rapidly cool the lattice. The faster the energy input, the even faster is the cooling due to sublimation. Thus, upon a rapid laser pulse there is a short spike of fairly cold evaporated guest molecules. (See computational results below.) To be sure, those guest molecules which have not escape during the sublimation spike and remain embedded in the matrix can get hotter and be thermally degraded. These molecules do no, however, get into the gas phase, since the lattice is now not as hot and the rate of sublimation is far slower.

The critical experimental parameters identified by the qualitative analysis of the mechanism are clear: (i) Because the guest molecules are embedded within a lattice of, typically much smaller, host molecules the guest concentration should be as low as possible to minimize energy transfer from the host. (ii) The laser power input and the host bulk parameters should be chosen so as to reach the threshold time for effective volatilization as rapidly as possible.

\section{Kinetic model}

To treat both infrared and UV excitation on a common footing we measure time $t$ in units of the halfwidth $\tau_{\mathrm{p}}$ of the laser pulse $\tau=t / \tau_{\mathrm{p}}$. It should be borne in mind that for the typical case of a $\mathrm{CO}_{2}$ laser $\tau_{\mathrm{p}}$ is nearly three orders of magnitude longer than for a UV pulse. The laser power input is, per unit matrix volume

$P_{\text {in }}=\alpha E_{0} \pi^{-1} \exp \left[-\left(\tau-\tau_{0}\right)^{2}\right]$.

Here, $\alpha$ is the absorption coefficient of the matrix, $E_{0}$ is the energy of the laser pulse per unit area (the fluence) and $\tau_{0}$ is the time at peak power of the pulse which we take to be Gaussian.

The UV laser input is absorbed by electronic excitation of the host molecules which is rapidly quenched by internal conversion. Some molecules can fluoresce but the quantum yield $\phi$ will be small. If desired this correction could be introduced into 
eq. (1) by multiplying the right-hand side by $1-\phi$. The infrared laser light is directly absorbed as vibrational excitation (of the host, guest and of any metallic particles). Let $H, L$ and $G$ be the mean energy content per unit volume of the single component of the hosts, lattice and guests. We claim that for typical situations (e.g. protein molecules in an nicotinic acid matrix )

$H=G \quad$ at thermal equilibrium .

The proof of (2) is based on the equipartition theorem and on the empirical observation that for peptides the number, $s$, of vibrational degrees of freedom (DOFs) is proportional (about $1 / 7$ th) to the molecular weight. The number of DOFs per unit volume is then proportional to $\left(N_{\mathrm{A}} / V\right) M$, where $V$ is the molar volume and $M$ the molecular weight and $N_{\mathrm{A}}$ is Avogadro's number. But the host and guest have comparable densities $\rho=M / V$.

In the kinetic equations for energy redistribution, the rates are then simply proportional to the energy differences

$$
\begin{aligned}
& \mathrm{d} H / \mathrm{d} \tau=x P_{\mathrm{in}}-\kappa_{\mathrm{HL}}(H-L)-\kappa_{\mathrm{HG}}(G-L), \\
& \mathrm{d} L / \mathrm{d} \tau=\kappa_{\mathrm{HL}}(H-L)-\kappa_{\mathrm{LG}}(L-G)-P_{\text {out }}, \\
& \mathrm{d} G / \mathrm{d} \tau=(1-x) P_{\mathrm{in}}+\kappa_{\mathrm{HG}}(H-G)+\kappa_{\mathrm{LG}}(L-G) .
\end{aligned}
$$

Here $x$ is the volume fraction of host molecules in the matrix $((1-x) \ll 1)$ and the $\kappa$ 's are rate coefficients (but where the unit of time is duration of the laser pulse). $P_{\text {out }}$ is the energy loss per unit time due to sublimation and strain and is further discussed below. We take the $\kappa$ 's to be of exponential gap type [9-11] as follows: for the host-lattice transfer

$\kappa_{\mathrm{HL}}=\tau_{\mathrm{p}} \nu_{\mathrm{H}} \exp \left(-\xi_{\mathrm{HL}}\right)$,

where $\xi$ is the adiabaticity parameter. Due to the anharmonicity of the host vibrational spectrum the transfer rate could be faster [12]. However, already with (6), the host-lattice exchange is so much faster than any other process that for all practical purposes the host and lattice are equilibrated on the timescale of real interest to us here. This will be quite evident from the computational examples below.

For the host-guest vibrational energy exchange we take into consideration that one guest molecule can be coupled to many host molecules. Note, however, that by the same argument (i.e. the larger volume of the guest molecule), there are more DOFs per guest molecule. The exponential gap rate is to be used per vibrational mode. On a per volume basis (assuming comparable densities) there are as many vibrational DOFs in the guest and host. Hence

$\kappa_{\mathrm{HG}}=\tau_{\mathrm{p}} C_{\mathrm{G}} \nu_{\mathrm{G}} \exp \left(-\xi_{\mathrm{GH}}\right)$,

where $C_{\mathrm{G}}$ is the volume fraction, $(1-x) / x, 1-x \ll 1$, of guest molecules in the matrix.

The adiabaticity parameter in $(6 \mathrm{~b})$ is similar to that used for a guest molecule physisorbed on a host in ref. [9]. Here it is the parameters of the hydrogen bond coupling of the guest and host that are to be used (rather than the physisorption bond parameters used in ref. [9]). Explicitly

$\xi_{\mathrm{GH}}=(D \Delta E)^{1 / 2} / \hbar \nu$,

where $D$ is the bond encrgy of the hydrogen bond and $\nu$ is its frequency. $\Delta E$ is the vibrational energy mismatch between guest and host.

The result ( $6 \mathrm{~b}$ ) is so central to our conclusions that we reiterate the derivation. Let $e_{\mathrm{H}}$ be the mean energy per host molecule. A particular host molecule is in direct contact with the fraction $C_{\mathrm{G}}$ of guest molecules $\left(C_{\mathrm{G}} \approx n_{\mathrm{G}} V_{\mathrm{G}} / n_{\mathrm{H}} V_{\mathrm{H}}\right.$ since $1-x \ll 1$ and $n$ is the number of molecules per unit volume of the matrix. As before, $V$ is the molar volume). Hence, the rate of change of $e_{\mathrm{H}}$ due to direct transfer to the guest is $\mathrm{d} e_{\mathrm{H}} / \mathrm{d} \tau=\kappa_{\mathrm{HG}} e_{\mathrm{H}}$. The rate of energy uptake by one guest molecule is $\mathrm{d} e_{\mathrm{G}} / \mathrm{d} \tau=\left(M_{\mathrm{G}} / M_{\mathrm{H}}\right) \mathrm{d} e_{\mathrm{H}} / \mathrm{d} \tau$. The kinetic equations follow upon computing on a per unit volume rather than on a per molecule basis, e.g. $H=n_{\mathrm{H}} e_{\mathrm{H}}$. The required changes if the guest and host are not of comparable densities should be clear from the derivation. The reasoning leading to the rate of lattice to guest coupling is similar. $\kappa_{\mathrm{LG}}$ is defincd by (6a) with $\nu_{\mathrm{L}}$ replacing $\nu_{\mathrm{H}}$. Since however $\nu_{\mathrm{L}}<\nu_{\mathrm{H}}$, this is a secondary route for energy transfer to the guest.

The laser pulse duration, $\tau_{\mathrm{p}}$, appears as a pre-exponential factor in (6) due to the use of reduced time which makes the power input depend on the laser fluence $E_{0}$, cf. eq. (1). For a given fluence, longer lasting pulses (e.g. in the infrared) lead to more effective energy redistribution. Other things being equal, the shorter $\tau_{\mathrm{p}}$ the lower is the energy content of the guest molecules.

Thermal degradation of the guest molecules is monitored by assuming an Arrhenius-type rate con- 
stant. The fraction of degraded molecules is then $1-\exp \left[-\kappa\left(T_{\mathrm{G}}\right) \tau\right]$ with

$\kappa\left(T_{\mathrm{G}}\right)=\tau_{\mathrm{p}} \nu_{\mathrm{G}} \exp \left(-D_{\mathrm{G}} / k T_{\mathrm{G}}\right)$

and $k T_{\mathrm{G}}$ is the mean energy per guest vibrational DOF. The threshold energy for degradation, $D_{\mathrm{G}}$, is taken at a quite low value so as to include isomcrization.

There are two mechanisms that we consider for draining energy out: sublimation and fragmentation. The rate of energy loss by sublimation is, per unit volume of the matrix

$P_{\text {out }}=\alpha\left(\Delta H_{\text {sub }} / N_{\mathrm{A}}\right) \rho_{\text {surf }} \nu_{\text {sub }} \exp \left(-\Delta H_{\text {sub }}\right) / k T_{\mathrm{L}}$.

$\Delta H_{\text {sub }}$ is the heat of sublimation per mol, $\rho_{\text {surf }}$ is the surface density of host molecules and $\nu_{\text {sub }}$ is the Arrhenius pre-exponential frequency which is expected [11] to be somewhat higher than the typical lattice frequency $\nu_{\mathrm{L}}$. One can also write (9) in terms of an equivalent pressure of gas phase host molecules [7].

It is the exponential increase of the rate of energy loss (9) with the mean energy per DOF of the lattice that is responsible for the "spike" in the rate of desorption which serves to rapidly cool the matrix. A short induction time after the laser onset, sublimation starts to accelerate. Once the laser energy input is over, sublimation cools the lattice and hence turns itself off.

The strain energy $S$ is computed [8] in terms of the stress and strain: $S=\frac{1}{2} \sigma \epsilon=\sigma^{2} / 2 K$ per unit volume, where $K$ is the bulk modulus. The thermally induced stress (assuming none to begin with), is related to the thermal expansion [8]

$\mathrm{d} \sigma / \mathrm{d} \tau=\frac{9}{2} K \alpha_{\mathrm{T}}\left(V_{\mathrm{L}} / C\right) \mathrm{d} L / \mathrm{d} \tau$.

$\alpha_{\mathrm{T}}$ is the volume thermal expansion coefficient and $C$ is the specific heat of the lattice and we neglected the rigidity modulus. We integrate (10) until $\sigma$ reaches the critical stress value $\sigma^{*}$. At that point we take the fragmentation to start. In section 3 we conclude that once the matrix fragments, guest molecules can escape. Hence whatever the energy content of the guest is at $\sigma=\sigma^{*}$, it is taken to be its initial value in the gas phase. In reality, some cooling is expected during the expansion stage of the fragmentation.

Values of critical stresses for many of the host ma- trices (which are often molecular crystals) could not be located by us in the literature. We have thus used the scaling [13]

$\sigma_{\mathrm{a}}^{*} / \sigma_{\mathrm{b}}^{*}=\left(K_{\mathrm{a}} \gamma_{\mathrm{a}} / K_{\mathrm{b}} \gamma_{\mathrm{b}}\right)^{1 / 2}$,

where $\gamma$ is the surface tension and subscripts refer to the particular solid.

The model could be improved upon in several ways. The energy coupling of laser and solid can be better handled, including spatial and temporal effects $[14,15]$. Other mechanisms, in particular the role of electronic excitation [16] and the importance of ablation [17] need be accounted for. The scaling of the energy transfer rate, eq. (7), could arguably be with the surface rather than the volume ratio of guest to host and the distribution of vibrational frequencies (and their anharmonicity) rather than a single representative frequency could be used.

\section{Fragmentation}

We argue that upon fragmentation the host matrix yields a large number of quite small fragments (rather than a few large ones). Therefore, upon fragmentation, many of the embedded guest molecules are released into the gas phase.

We treat the fragmentation of the matrix just as we treated the laser induced fragmentation of a single molecule [18]. For simplicity we consider a pure host matrix.

The first step is to list the possible fragments. We do not allow dissociation of the host molecules. The fragments are then clusters of $N$ hosi molecules, $N=1$, $2, \ldots$. Let $X_{N}$ be the number of clusters of $N$ host molecules. Then, conservation of host molecules implies

$\sum N X_{N}=N_{0}$,

where $N_{0}$ is the number of host molecules in the "hot spot". We similarly know the total energy

$\sum E_{N} x_{N}=E_{0}$,

where $E_{N}$ is the energy of one cluster of $N$ molecules. Note that the total number of fragments, $X$,

$X=\sum X_{N}$,

is not known to begin with.

The constraints (12) and (13) are just those used 
in the fragmentation problem before [18] so that the distribution $X_{N}$ which satisfies the constraints and is of maximal entropy is

$X_{N}=X \exp \left(-\beta \mu N-\beta G_{N}\right)$.

Here, $\exp \left(-\beta G_{N}\right) \equiv Q_{N}$ is the partition function of the cluster of size $N$. The values of the Lagrange multipliers $\mu$ and $\beta$ can be determined in terms of the given $N_{0}$ and $E_{0}$.

To provide a connection with familiar results, say the host molecules are monoatomic and contain no thermal energy. Then, $G_{1}=0$ and $X_{1} / X=\exp (-\beta \mu)$ so that (15) can be written as

$X_{N}=X\left(X_{1} / X\right)^{N} \exp \left(-\beta G_{N}\right)$.

(16) is just the Frenkel distribution (ref. [19] section VII.3, see also, e.g. ref. [20]) of droplet sizes.

The $N$ dependence of $G_{N}$ governs the overall shape of the distribution (15). The essential point is that for materials of finite surface tension $\gamma, G_{N}$ versus $N$ has a maximum so that the distribution of clusters has a minimum and, when as is typically the case, $\beta \mu>0, X_{1}$ is largest of the small sized clusters.

The maximum in $G_{N}$ versus $N$ is the familiar high vapor pressure of small clusters. In a simple liquid drop model

$G_{N}=-N \Delta G+4 \pi \gamma\left(3 N V / 4 \pi N_{\mathrm{A}}\right)^{2 / 3}$,

where $V$ is the molar volume of the solid and $\Delta G$ is the free energy of sublimation per molecule of the bulk solid. $\gamma$ is the surface tension and $N_{\mathrm{A}}$ is Avogadro's number. It should, however, be clearly recognized that (17) is an oversimplification and neglects such important factors as the internal energy content of the cluster. We intend to return to this problem in a future publication.

\section{Computational study}

Using realistic, typical values for the laser and material parameters, the kinetic equations were integrated. The numerical results provide a quantitative illustration of the essential points of the model.

The sublimation spike, for UV irradiation of matrices with a low heat of sublimation, is clearly evident in fig. 1. The rapid onset of sublimation is due to the Gaussian profile of the laser power, also shown
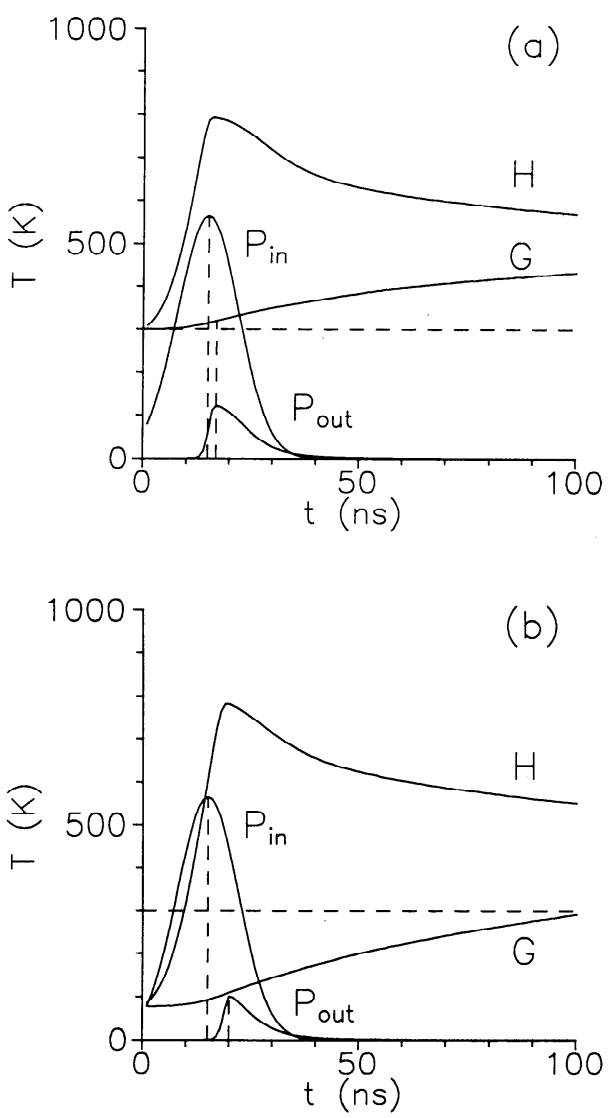

Fig. 1. Energy pathways for UV laser irradiation of a nicotinic acid matrix containing a $10^{-4}$ volume fraction of $M_{\mathrm{G}}=10^{5}$ protein. Initial conditions (a) room temperature (b) liquid nitrogen temperature. $P_{\text {in }}$ and $P_{\text {out }}$ are the laser power input and sublimation power output per unit matrix area. Note that the mean energy per guest degree of freedom $\left(\approx 7 V_{\mathrm{G}} G / M_{\mathrm{G}}\right)$ never gets very high during the sublimation stage. See text for definition of symbols and further details.

in the figure. Note the induction time before the sublimation is "turned on". Once the laser is off, the evaporation causes cooling of the lattice and turns itself off. The guest molecules are desorbing essentially only in a time window of width $\tau_{\mathrm{p}}$, which is $\approx 10$ ns for a quadrupled Nd:YAG laser. The lower the volume ratio $C_{\mathrm{G}}$ of the guest, the weaker the coupling to the host, cf. eq. (6b) $C_{\mathrm{G}}=10^{-4}$ in fig. 1 . We have verified that for higher values of $C_{\mathrm{G}}$ the thermal degradation is more extensive than shown in fig. 1 . Note that $C_{\mathrm{G}}$ is governed both by the inherent molar volumes of the guest and host and by the exper- 
imentally controllable mole fraction of the guest.

It is clearly advantageous to use host molecules of low sublimation temperature and poorer frequency matching with the guest. (In fig. 1 we use the parameters of the most common host, nicotinic acid, for which $\nu_{\mathrm{G}} \approx \nu_{\mathrm{H}}$ )

Fig. 1 compares the vaporization from an initially room temperature matrix and one that was cooled to liquid nitrogen temperature. The sublimation spike is practically identical but the low initial value of the guest energy per DOF prevents its getting warm dur-
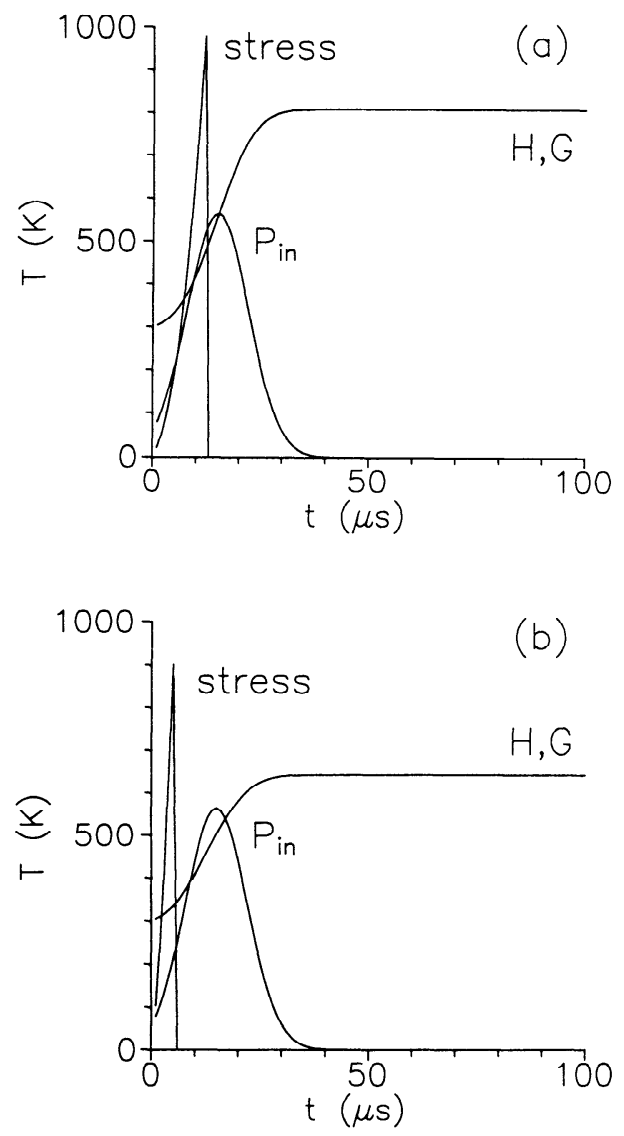

Fig. 2. Temporal evolution for a $\mathrm{CO}_{2}$ laser pulse. The temperature scale is for the guest, $G$. The pulse profile versus time is labelled as $P_{\text {in. }}$. The stress curve is $1000\left(\sigma / \sigma^{*}\right)$, where $\sigma^{*}$ is the critical stress. At $\sigma=\sigma^{*}$ the matrix fragments. (a) High guest concentration, $C_{\mathrm{G}}=0.5$, in an alkali-halide host, $\sigma^{*}=10^{5} \mathrm{~N} \mathrm{~cm}^{-2}$, $K=5 \times 10^{6} \mathrm{~N} \mathrm{~cm}^{-2}$. (b) Same as (a) but with twice the values of $K$ and $\alpha_{\mathrm{T}}$ (as would be the case in the presence of metallic powder). ing the sublimation. Cooling the matrix prior to UV irradiation is clearly very beneficial.

On the timescale shown there is no significant fragmentation. This is to be expected for a sonic stress whose maximal propagation velocity (lattice constant $\nu_{\mathrm{L}}$ ) will not exceed a few angstrom per picosecond.

Fig. 2 shows similar results for $\mathrm{CO}_{2}$ laser infrared heating. Note first the much longer timescale as compared to fig. 1. For typical host materials as used in the infrared (e.g. alkali halides, sugars [5] ), there is practically no evaporation but there is extensive fragmentation of the matrix. Here it is the rapid rise of the stress that is responsible for volatilization via matrix fragmentation of undegraded guest molecules. Note, however, that for infrared heating, the energy content of the guests is significantly higher than for typical conditions in the UV-type experiments, cf. fig. 1. Host matrices with high absorption and low critical stress would work best.

Fig. 3 shows the fraction of degraded guest molecules for both IR and UV lasers. The longer acting IR pulse leads to inevitable higher losses of guest molecules. Note that the computation is for the guest molecules which remain embedded in the host. Even

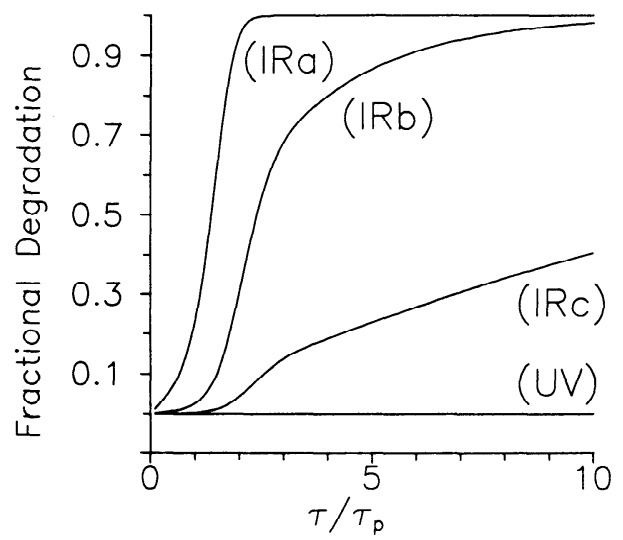

Fig. 3. Fractional degradation of the guest biomolecules versus the reduced time $\tau=t / \tau_{\mathrm{p}}$. The IR values are for saccharides as hosts $\left(\sigma^{*}=10^{4} \mathrm{~N} \mathrm{~cm}^{-2}\right)$ at three values of the threshold energies for thermal degradation (a) $=54 \mathrm{~kJ} \mathrm{~mol}^{-1}$, (b) $=60 \mathrm{~kJ} \mathrm{~mol}^{-1}$ and (c) $=66 \mathrm{~kJ} \mathrm{~mol}^{-1}$, respectively. For the UV excitation (case of fig. 1a) there is practically no degradation. Note, however, that at most by $\tau \approx 3$ the volatilization is over, so that for longer times the results refer to the biomolecules which remain embedded in the matrix. 
in the IR, using materials of high thermal expansion and low critical stress can lead to extensive volatilization for $\tau<1$ and hence to undegraded molecules in the gas phase.

\section{Concluding remarks}

A simplistic model based on a competition between intermolecular energy transfer and loss of material to the gas phase was discussed. For realistic laser and material parameters it is concluded that the observed volatilization of undegraded biomolecules can be rationalized on this basis.

\section{Acknowledgement}

We thank Professor M.J. Berry, Professor J. Grotemeyer and Professor E.W. Schlag for useful discussions and comments. This work was supported by the US-Israel Binational Science Foundation (BSF) Jerusalem, Israel. The Fritz Haber Research Center is supported by the Minerva Gesellschaft für die Forschung, mbH, Munich, BRD.

\section{References}

[1] J. Grotemeyer and E.W. Schlag, Accounts Chem. Res. 22 (1989) 399.

[2] M. Karas, U. Bahr, A. Ingendoh and F. Hillenkamp, Angew. Chem. Intern. Ed. Engl. 28 (1989) 760.

[3] M. von Allmen, Laser-beam interactions with materials, physical principles and applications (Springer, Berlin, 1987).

[4] K. Tanaka, H. Waki, Y. Ido, S. Akita, Y. Yoshida and T. Yoshida, Rapid Commun. Mass Spectrom. 2 (1988) 151; M. Karas and F. Hillenkamp, Anal. Chem. 60 (1988) 2299;
M. Karas, U. Bahr and F. Hillenkamp, Intern. J. Mass Spectrom. Ion Processes 92 (1989) 231;

J. Grotemeyer and E.W. Schlag, Org. Mass Spectrom. 23 (1988) 388;

M. Salehpour, I. Perera, J. Kjellberg, A. Hedin, M.A. Islamian, P. Hakansson and B.U.R. Sundqvist, Rapid Commun. Mass Spectrom. 3 (1989) 259;

R.W. Nelson, M.J. Rainbow, D.E. Lohr and P. Williams, Science 246 (1989) 1585.

[5] R.C. Beavis, J. Lindner, J. Grotemeyer and E.W. Schlag, Chem. Phys. Letters 146 (1988) 310;

R.C. Beavis, J. Grotemeyer and E.W. Schlag, Z. Naturforsch. 43a (1988) 1083.

[6] R.C. Beavis and B.T. Chait, Rapid Commun. Mass Spectrom. 3 (1989) 233.

[7] A. Vertes, R. Gijbels and R.D. Levine, to be published.

[8] L.H. Adams and R.M. Waxler, Temperature-induced stresses in solids of elementary shape', NBS Monograph 2, US Department of Commerce, June 1960.

[9] R.N. Zare and R.D. Levine, Chem. Phys. Letters 136 (1987) 593.

[10] G.E. Ewing, J. Phys. Chem. 91 (1987) 4662.

[11] R.D. Levine and R.B. Bernstein, Molecular reaction dynamics and chemical reactivity (Oxford Univ. Press, Oxford, 1987).

[12] B. Fain and S.H. Lin, Chem. Phys. Letters 114 (1985) 497; 157 (1989) 233.

[13] J.C. Jaeger, Elasticity, fracture and flow (Wiley, New York, $1956)$ p. 85.

[14] M. Bassin, Physical processes in laser-materials interactions, ed. M. Bertolotti (Plenum Press, New York, 1980).

[15] A. Vertes, P. Juhasz, M. DeWolf and R. Gijbels, Intern. J. Mass Spectrom. Ion Processes 94 (1989) 63.

[16] R.E. Johnson, Intern. J. Mass Spectrom. Ion Processes 78 (1987) 357.

[17] E. Sutcliffe and R. Srinivasan, J. Appl. Phys. 60 (1986) 3315;

H. Masuhara, S. Eura, H. Fukumura and A. Itaya, Chem. Phys. Letters 156 (1989) 446.

[18] J. Silberstein and R.D. Levine, Chem. Phys. Letters 74 (1980) 6; J. Chem. Phys. 75 (1981) 5735.

[19] J. Frenkel, Kinetic theory of liquids (Dover, New York, 1955).

[20] D.H. Rasmussen, M.R. Appleby, G.L. Leedom and S.V. Babu, J. Cryst. Growth 64 (1983) 229. 